\title{
Current Status in the Treatment of Acute Cholecystitis Patients Receiving Antithrombotic Therapy: Is Endoscopic Drainage Feasible?- A Systematic Review
}

\author{
Ryota Sagami', Kenji Hayasaka', Hidefumi Nishikiori', Hideaki Harada ${ }^{1}$ and Yuji Amano ${ }^{3}$ \\ ${ }^{1}$ Department of Gastroenterology, New Tokyo Hospital, Chiba, ${ }^{2}$ Department of Gastroenterology, Oita San-ai Medical Center, Oita, \\ ${ }^{3}$ Department of Endoscopy, New Tokyo Hospital, Chiba, Japan
}

The bleeding complication risk of surgery or percutaneous transhepatic gallbladder drainage (PTGBD) may increase in patients with acute cholecystitis receiving antithrombotic therapy (ATT). Endoscopic gallbladder drainage (EGBD) may be recommended for such patients. English articles published between 1991 and 2018 in peer-reviewed journals that discuss cholecystectomy, PTGBD, and EGBD in patients with ATT or coagulopathy were reviewed to assess the safety of the procedures, especially in terms of the bleeding complication. There were 8 studies on cholecystectomy, 3 on PTGBD, and 1 on endoscopic transpapillary gallbladder drainage (ETGBD) in patients receiving ATT. With respect to EGBD, 28 studies on ETGBD (including 1 study already mentioned above) and 26 studies on endoscopic ultrasound-guided gallbladder drainage (EUS-GBD) were also analyzed. The overall bleeding complication rate in patients with ATT who underwent cholecystectomy was significantly higher than that in patients without ATT (6.5\% [23/354] vs. 1.2\% [26/2,224], $p<0.001)$. However, the bleeding risk of cholecystectomy and PTGBD in patients receiving ATT was controversial. The overall technical success, clinical success, and bleeding complication rates of ETGBD vs. EUS-GBD were $84 \%$ vs. $96 \%$ ( $p<0.001), 92 \%$ vs. $97 \%$ ( $p<0.001$ ), and $0.65 \%$ vs. $2.1 \%$ ( $p=0.005)$, respectively. One patient treated with ETGBD experienced bleeding complication among 191 patients with bleeding tendency. ETGBD may be an ideal drainage procedure for patients receiving ATT from the viewpoint of bleeding, although EUS-GBD is also efficacious. Clin Endosc 2020;53:176-188

Key Words: Acute cholecystitis; Antithrombotic therapy; Endoscopic transpapillary gallbladder drainage; Endoscopic ultrasoundguided gallbladder drainage; Percutaneous transhepatic gallbladder drainage

\section{INTRODUCTION}

Acute cholecystitis is one of the most common biliary diseases and sometimes presents as an emergency condition, possibly inducing severe complications when appropriate drainage is not carried out. Therefore, gallbladder drainage at an early stage is essential in patients with severe cholecystitis

Received: September 9, 2019 Revised: October 11, 2019

Accepted: October 12, 2019

Correspondence: Ryota Sagami

Department of Gastroenterology, New Tokyo Hospital, 1271 Wanagaya, Matsudo, Chiba 270-2232, Japan

Tel: +81-47-711-8700, Fax: +81-47-392-8718, E-mail: sagami1985@yahoo.co.jp ORCID: https://orcid.org/0000-0003-4961-3521

(c) This is an Open Access article distributed under the terms of the Creative Commons Attribution Non-Commercial License (http://creativecommons.org/ licenses/by-nc/3.0) which permits unrestricted non-commercial use, distribution, and reproduction in any medium, provided the original work is properly cited. if multiple organ dysfunction or severe local inflammation is found. ${ }^{1}$ Cholecystectomy remains the gold standard management approach for patients with acute cholecystitis. Since the introduction of laparoscopic cholecystectomy (LC), minimally invasive surgery has been commonly performed in patients with acute cholecystitis, compared with open cholecystectomy (OC). ${ }^{2}$ However, these surgical procedures may result in increased mortality in elderly patients and/or in those with multiple severe comorbidities. ${ }^{3}$ In such cases, percutaneous transhepatic gallbladder drainage (PTGBD) is often performed instead of surgery. PTGBD is an effective clinical method of primary drainage with a high technical success rate. ${ }^{4}$ However, PTGBD is generally prohibited in patients with massive ascites, anatomically inaccessible gallbladders, and a high risk of self-removal of the drainage tube, and it is especially risky in patients with a bleeding tendency. ${ }^{5}$ In addition, PTGBD is 
associated with adverse events including bleeding or bile leakage, a high recurrence rate, and fistula formation after longterm stent placement and stent displacement. ${ }^{4,5}$

Considering these disadvantages, endoscopic gallbladder drainage (EGBD), including endoscopic transpapillary gallbladder drainage (ETGBD) and endoscopic ultrasound-guided gallbladder drainage (EUS-GBD), may be more useful in such patients. ETGBD via a nasobiliary catheter was first reported, and ETGBD via an internal plastic stent was reported a year later. ${ }^{6}$ This method, through endoscopic retrograde cholangiopancreatography (ERCP), was reported to be effective for gallbladder drainage with relatively high technical and clinical success rates, ${ }^{7}$ and to be an option for patients in whom PTGBD treatment is difficult. ${ }^{5}$ The next endoscopic method reported was EUS-GBD, ${ }^{8}$ which is a stent placement method under EUS guidance from the antral, duodenal, or jejunal lumen to the gallbladder, and showed higher technical and clinical success rates than expected. ${ }^{9}$ Currently, EGBD is established as the third choice for the treatment of acute cholecystitis, following LC as the first choice and PTGBD as the second choice, owing to the potential low risk of bleeding of endoscopic methods. Surgical and conservative treatments, including PTGBD or EGBD, for acute cholecystitis in patients with antithrombotic agents will likely become common, as the number of elderly patients with cardiovascular and cerebrovascular diseases is increasing remarkably.

Therefore, the latest guidelines recommended these drainage methods for patients receiving antithrombotic therapy (ATT) or those with a bleeding tendency. ${ }^{10}$ However, there have been few studies comparing these surgical and percutaneous techniques with EGBD techniques, particularly in terms of bleeding complications, in patients receiving ATT or with a bleeding tendency. To the best of our knowledge, this is the first review on EGBD in patients receiving ATT.

\section{MATERIALS AND METHODS}

From January to February 2019, we searched the PubMed and Google Scholar databases (restricted to articles written in English and published between 1991 and 2018) to retrieve internationally accepted English abstracts of articles on LC and PTGBD in patients receiving ATT or with coagulopathy, and articles about EGBD. The following key words were used for the search: antithrombotic therapy, antiplatelet, anticoagulant, warfarin, clopidogrel, aspirin, bleeding, hemorrhage, endoscopic gallbladder drainage, endoscopic ultrasound-guided gallbladder drainage, endoscopic transpapillary gallbladder drainage, percutaneous transhepatic drainage, cholecystectomy, cholecystostomy, laparoscopic, and cholecystitis.
Articles published in peer-reviewed journals as full articles were included in this review. The types of articles included randomized controlled trials (RCTs), prospective studies, and retrospective cohort studies that examined 5 cases or more. Guidelines, review articles, and case series/reports were not included in this analysis. RCTs and prospective studies were of a relatively high evidence level; however, retrospective studies may have had potential selection bias. The quality of each study was assessed depending on the study design, and eligible articles were identified. The full texts of all articles were read by 2 independent reviewers (RS and YA). Complete data were extracted from each study, including the year of publication, type of procedure (LC, PTGBD, ETGBD, or EUS-GBD), study design, sample size, age, sex, and complication rate (including bleeding complications), which were also analyzed in patients receiving ATT or with a bleeding tendency. In addition, the technical and/or clinical success rate and the recurrence rate were analyzed in cases of ETGBD and EUS-GBD. Data were extracted into an Excel spreadsheet for further analysis, including statistical analysis.

\section{Statistical analysis}

Categorical variables are expressed as proportions and analyzed with Fisher's exact test or the chi-square test, and $p<0.05$ was considered statistically significant. All data analyses were conducted with SPSS version 24.0 (IBM Co., Armonk, NY, USA).

\section{Definitions}

The bleeding complication rate of each drainage procedure was analyzed in detail. Bleeding complication was defined as the presence of anemia (hemoglobin reduction of $2 \mathrm{~g} / \mathrm{dL}$ or more), requirement for a blood transfusion, and/or the need for another additional treatment for bleeding such as interventional radiological or surgical therapy. The technical success, clinical success, and recurrence rates were mainly evaluated in ETGBD and EUS-GBD cases. Technical success was defined as successful stent placement in the gallbladder through the cystic duct in ETGBD, and successful stent placement between the stomach or duodenal lumen and the gallbladder in EUS-GBD. Clinical success was defined as the improvement of cholecystitis after the first drainage procedure. Clinical success rate was defined as the proportion of patients with clinical success divided by the proportion of those with technical success. Minor symptoms, such as lowgrade fever and mild pain, which were not clinically relevant or conservatively improved, were not included as complications in the present analyses. In this review, early complication was defined as a complication that occurred within 5 days from the procedure, and late complication, a complication 
that occurred 6 or more days after the procedure. Recurrence rate was defined as the proportion of patients with recurrent acute cholecystitis among those with clinical success, excluding patients who died or underwent stent exchange or elective surgical cholecystectomy. Each rate was calculated considering the non-assessed cases.

Patients with a bleeding tendency were defined as those receiving antiplatelet agents and/or anticoagulants. Patients with moderate or severe liver disease who showed moderate abnormalities of prothrombin time-international normalized ratio (PT-INR) and/or platelet count, including those with end-stage liver disease requiring blood transplantation and those with leukemia and disseminated intravascular coagulation, were also included.

\section{RESULTS}

\section{Study collection}

Research article collection and screening were conducted from January 2019 to February 2019. A total of 8 studies on surgical cholecystectomy (mainly LC) ${ }^{11-18} 3$ studies on PTGBD, ${ }^{19-21}$ and 1 study on $\mathrm{EGBD}^{5}$ in patients receiving ATT were selected for data extraction and satisfied our inclusion criteria. As only 1 study on EGBD in patients receiving ATT was retrieved through the search method mentioned above, articles were additionally searched using the keywords "ETGBD" and "EUS-GBD". As a result, 27 studies on ETGBD $^{6,22-47}$ and 26 studies on EUS-GBD ${ }^{8,37,40,45,46,48-68}$ were newly found. Finally, 28 studies on ETGBD and 26 studies on EUS-GBD were evaluated in this review, which included some cases with bleeding tendency and ATT administration.

Three articles on the EUS-GBD technique were excluded because the procedure was converted from PTGBD, and the accurate therapeutic effects of EUS-GBD could not be evaluated in these cases.

\section{Gallbladder drainage in patients receiving ATT}

In the 12 studies that evaluated surgery and gallbladder drainage, patients receiving ATT, including aspirin and/or thienopyridine, were the focus of 3 studies. ${ }^{12,14,15}$ Only 1 study evaluated patients receiving anticoagulant therapy with heparin bridging, ${ }^{11} 1$ study evaluated patients receiving anticoagulant therapy and/or with coagulopathy, ${ }^{19}$ and the other studies $^{5,13,16-18,20,21}$ evaluated patients receiving antiplatelet agents and/or anticoagulants during surgery (LC or OC) or internal drainage (PTGBD or EGBD) without discontinuation of ATT (Table 1).

Overall, $13.7 \%(354 / 2,578)$ of patients receiving antithrombotic agents underwent LC. There were no significant dif- ferences between patients with continued and discontinued antiplatelet therapy (aspirin and/or thienopyridine) in intraoperative blood loss, operative time, conversion rate to open surgery, 30-day morbidity, or bleeding complications requiring blood transfusion. ${ }^{12,14,15}$ In some cases of cholecystectomy in patients receiving antiplatelet agents and/or anticoagulants, there were similar tendencies found in LC. ${ }^{13,16}$ On the other hand, 2 studies on cholecystectomy in patients receiving antiplatelet agents and/or anticoagulants reported that anemia was more frequently observed in patients who underwent emergency LC with continuation of ATT, ${ }^{17}$ and that treatment with multiple antiplatelet therapies and anticoagulation therapies was an independent predictor for postoperative bleeding complications. ${ }^{18}$ Moreover, in a study on LC, it was proven that patients receiving heparin bridging therapy had a significantly higher rate of surgical blood loss than those without anticoagulants. ${ }^{11}$ Overall, bleeding complications occurred in $6.5 \%(23 / 354)$ of patients with ATT and in 1.2\% $(26 / 2,224)$ of patients without ATT $(p<0.001)$.

With respect to PTGBD, 1 study in patients receiving anticoagulant therapy or with coagulopathy revealed that the cessation of ATT $24 \mathrm{~h}$ before the procedure or abnormal coagulation (INR $\geq 1.5$ or platelet count $\leq 50 \times 10^{9} / \mathrm{L}$ ) induced major complications related to PTGBD (hemorrhage requiring blood transfusion, sepsis and death directly related to the procedure, and abscess), although they did not occur in patients with normal coagulation, and that 132 patients receiving anticoagulant therapy and/or with coagulopathy had a bleeding complication rate of $1.5 \% .^{19}$ Shibasaki et al. ${ }^{20}$ reported that in PTGBD followed by elective LC, no bleeding complications were seen in 23 patients receiving ATT. On the other hand, Hamada et al..$^{21}$ analyzed 34,606 patients who underwent percutaneous transhepatic biliary drainage (PTBD) or PTGBD (23,375 PTBDs and 11,231 PTGBDs), including 1.5\% $(503 / 34,606)$ patients with continued antiplatelet and anticoagulant therapy, both of which were administered on the day of the procedure. The overall rate of severe bleeding complications requiring blood transfusion was 2.3\% (PTBD 2.5\% and PTGBD 1.6\%); however, it was $4 \%$ in patients with continued ATT in this study. In addition, continuation of antiplatelet agents was significantly associated with severe bleeding (4.7\%, $p=0.013$ ), whereas no significant difference was observed in the rate of bleeding complications in patients who discontinued antiplatelet agents $(p=0.517)$.

There was only 1 study on ETGBD in patients with ATT. In that study, no bleeding complications were seen in any of the 35 cases. $^{5}$

\section{Present status of EGBD: ETGBD and EUS-GBD}

EGBD consists of 2 different methods: transpapillary 


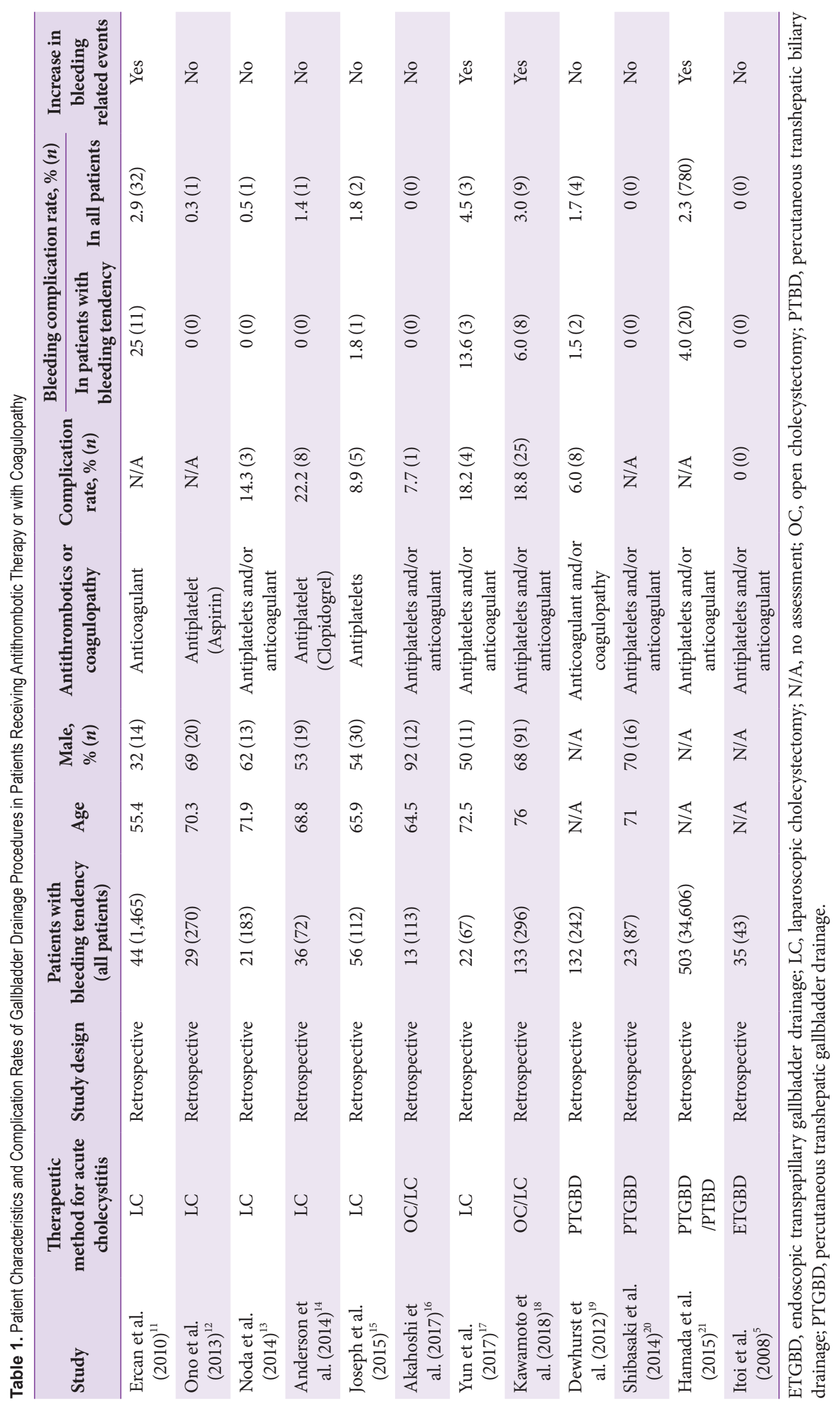


Ce cunncal enoscopry

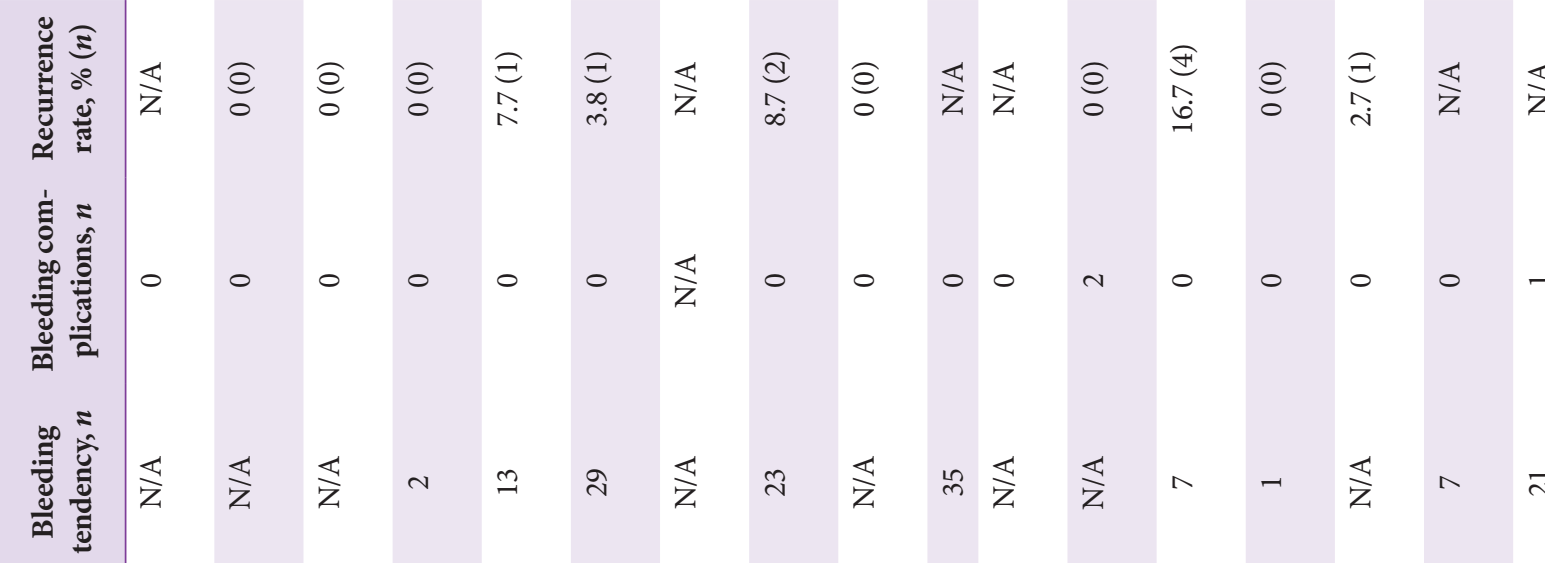

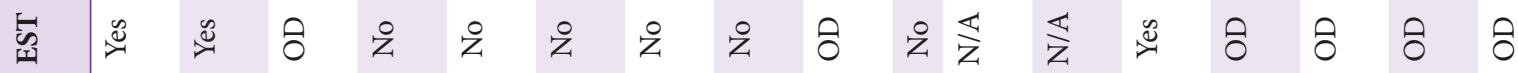

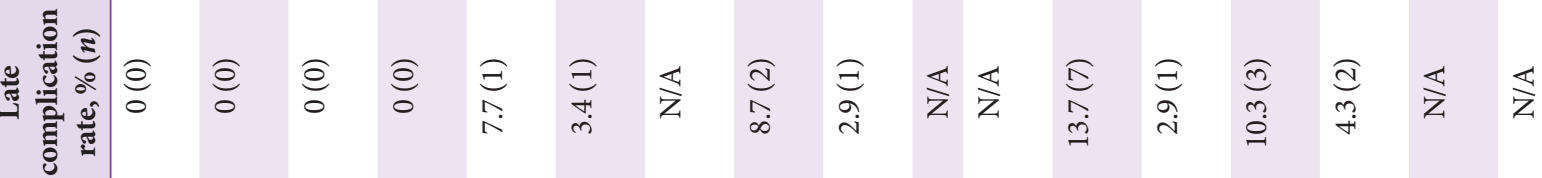

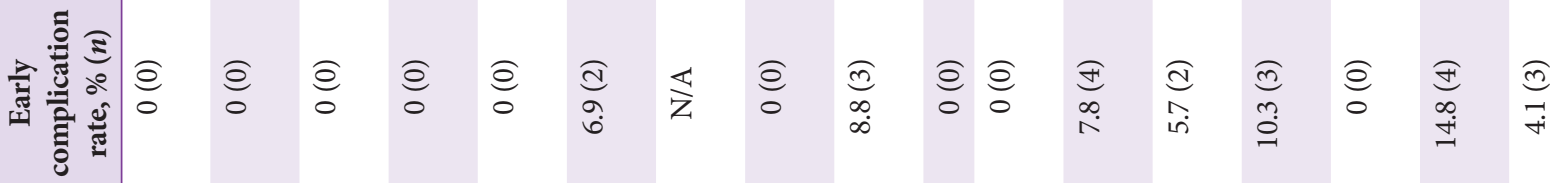

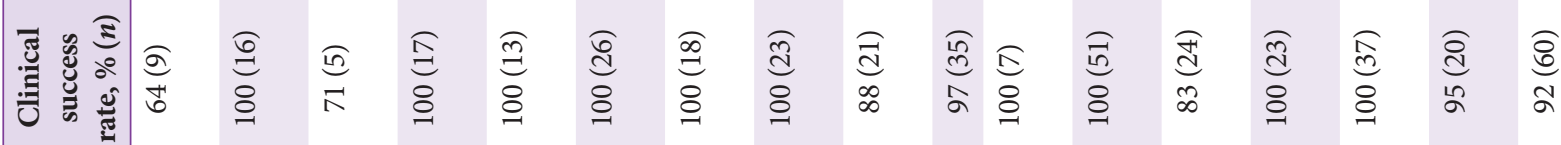

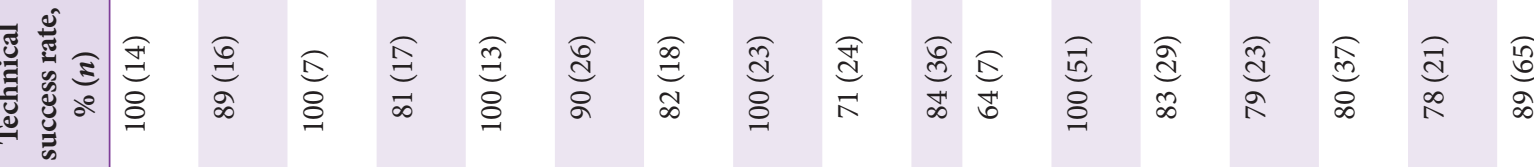

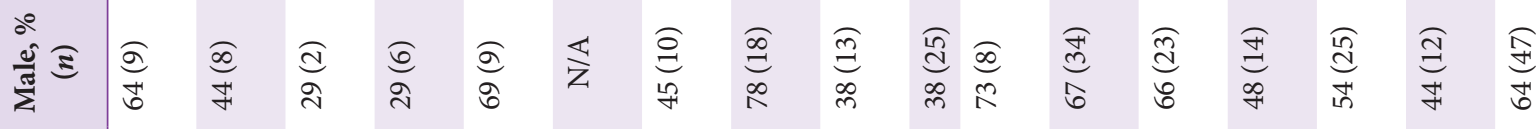

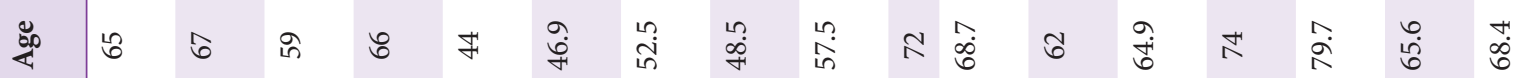

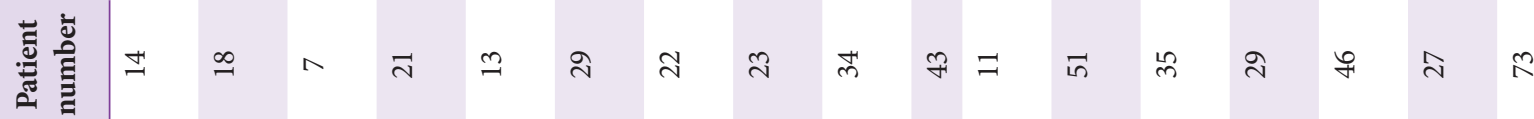

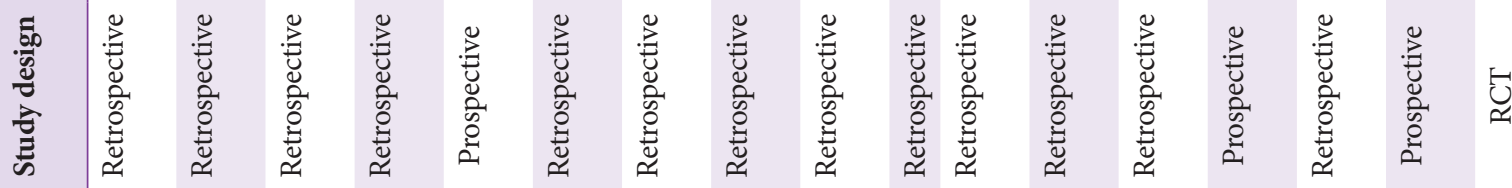

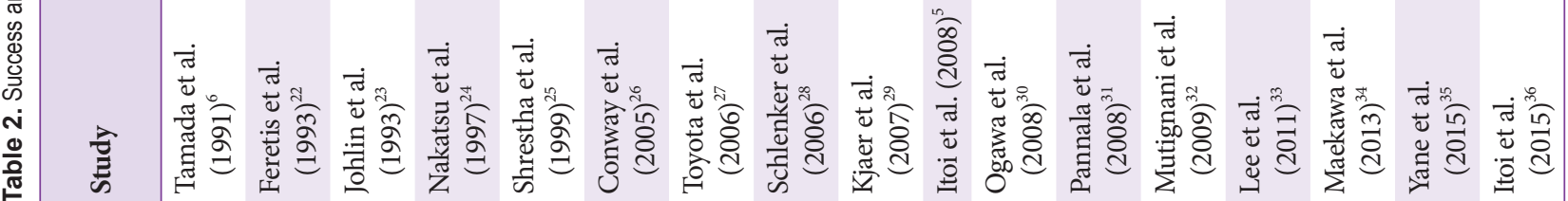




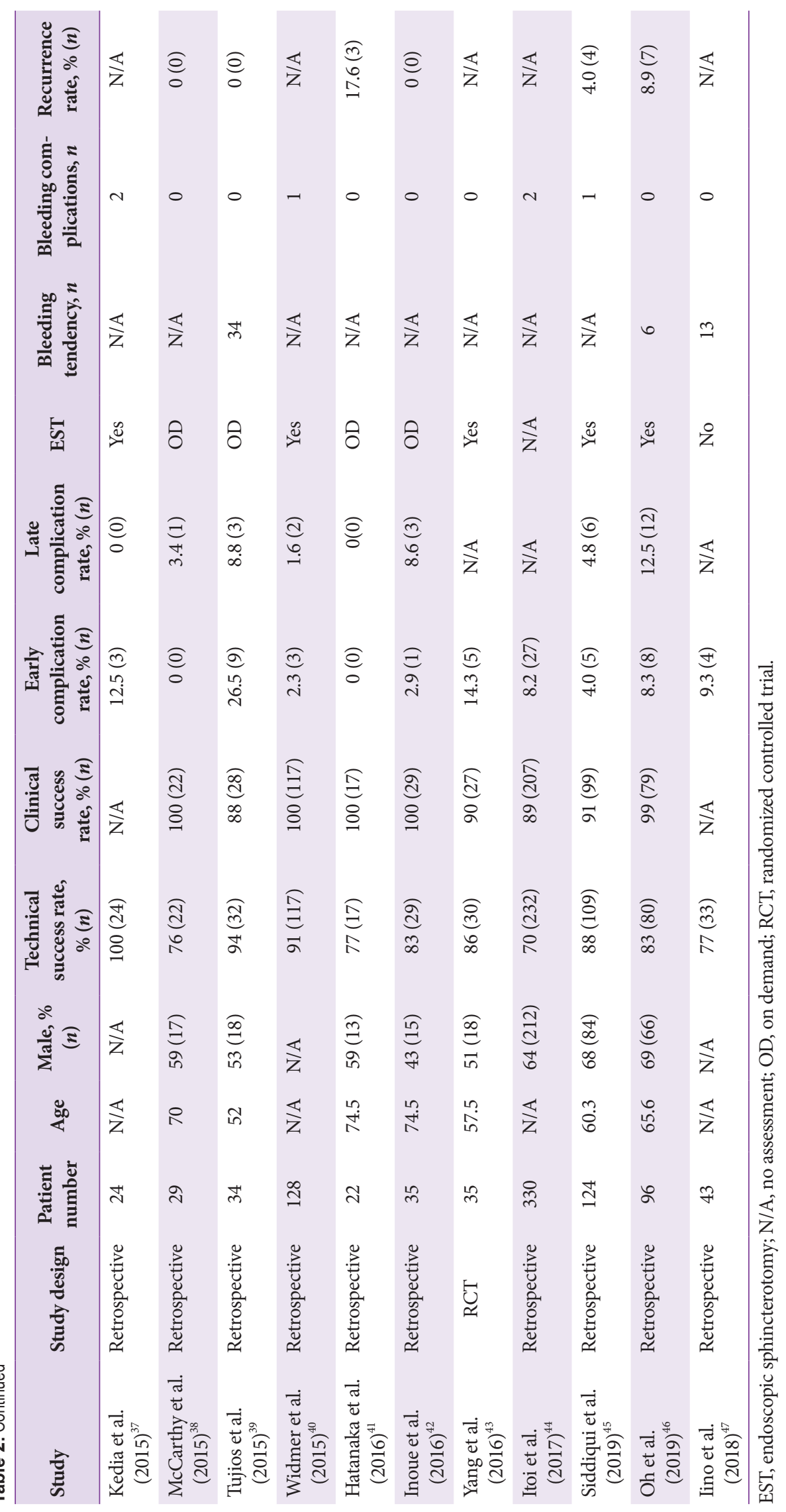


ETGBD and transmural EUS-GBD. ETGBD requires the ERCP procedure and placement of a plastic stent or nasobiliary catheter into the gallbladder through the duodenal papilla and the cystic duct. EUS-GBD requires EUS guidance, and approaches the gallbladder from the gastric antrum or the duodenum for the drainage procedure. Plastic stents (initially) or metal stents (more recently) are placed between the gallbladder lumen and the gastrointestinal lumen.

There were 28 studies $^{5,622-47}$ on ETGBD, including 2 RCTs, 3 prospective studies, and 23 retrospective studies (Table 2). Endoscopic sphincterotomy (EST) in the ETGBD procedure was classified as follows: performed (yes), not performed (no), or on demand. The enrolled patients were 22-95 years old, and $60 \%(706 / 1,172)$ were men. The technical and clinical success rates of ETGBD were $64 \%-100 \%$ and $64 \%-100 \%$, respectively. The early and late complication rates were $0 \%-26.5 \%$ and $0 \%-13.7 \%$, respectively. The recurrence rate was $0 \%-17.6 \%$. Overall, 13.7\% (191/1,396) of the patients had some type of bleeding tendency that could be confirmed. The overall technical and clinical success rates were $84 \%(1,176 / 1,396)$ and $92 \%(1,030 / 1,119)$, respectively. Early complications included pancreatitis due to the ERCP procedure, bleeding (especially after EST), choledocholithiasis, cystic duct perforation caused by the guidewire or the cannulation technique, cholangitis, sepsis, migration, and bile leakage. Late complications included pericholecystic fluid collection, cholangitis, duodenal perforation, sepsis, choledocholithiasis, stent migration, stent occlusion, hepatic abscess, and duodenal ulcer, and were treated conservatively. The early and late complication rates were $6.3 \%(86 / 1,374)$ and $5.5 \%(45 / 812)$, respectively. The bleeding complication rate was $0.65 \%(9 / 1,374)$. The overall recurrence rate was $2.2 \%(23 / 1,030)$.

Twenty-six studies ${ }^{8,37,40,45,46,48-68}$ on EUS-GBD, including 1 RCT, 4 prospective studies, and 21 retrospective studies, were analyzed (Table 3 ). The puncture sites were the duodenal bulb in 55\% (334/609), the antrum in 44\% (268/609), and the jejunum in $1 \%(7 / 609)$. The enrolled patients were $25-97$ years old, and 53\% (392/741) were men. The technical and clinical success rates were $85 \%-100 \%$ and $92 \%-100 \%$, respectively. The early and late complication rates were $0 \%-28.6 \%$ and $0 \%-15.4 \%$, respectively. The recurrence rate was $0 \%-8.3 \%$. Eighteen patients had some kind of bleeding tendency. The technical and clinical success rates were $96 \%$ (729/758) and 97\% (704/723), respectively. Early complications included pneumoperitoneum, peritonitis, perforation, migration, bile fluid collection, bleeding, pancreatic infection, sepsis, and bile leak, whereas the major early complications were pneumoperitoneum and peritonitis. Late complications included stent migration, stent occlusion, abscess, and bleeding. The early and late complication rates were 7.0\% (53/758) and 4.4\%
(33/749), respectively. The bleeding complication rate was $2.1 \%$ (16/749). The overall recurrence rate was 3.0\% (21/703). These overall results of the reviewed ETGBD and EUS-GBD procedures are shown in Fig. 1.

\section{Comparison of ETGBD and EUS-GBD}

The technical and clinical success rates of ETGBD vs. EUSGBD were $84 \%(1,176 / 1,396)$ vs. $96 \%$ (729/758), $p<0.001$ and $92 \%(1,030 / 1,119)$ vs. $97 \%$ (704/723), $p<0.001$, respectively. These results may suggest that EUS-GBD is easier to perform successfully and is more clinically useful than ETGBD. The early and late complication rates of ETGBD vs. EUS-GBD were $6.3 \%(86 / 1,374)$ vs. $7.0 \%$ (53/758), $p=0.522$ and $5.5 \%$ $(45 / 812)$ vs. $4.4 \%$ (33/749), $p=0.352$, respectively. The most frequent early complication of ETGBD was pancreatitis, which occurred at a rate of $2.3 \%(32 / 1,374)$ in this study. In EUSGBD, pneumoperitoneum, peritonitis, and bile leak were the most frequent, and the rate of early complication was 3.8\% (29/758). The most frequent late complications of ETGBD and EUS-GBD were migration and occlusion of the drainage tube, which possibly induced recurrent cholecystitis or cholangitis, and the rate was 2.8\% (23/812) for ETGBD and 3.0\% (23/749) for EUS-GBD. There were no statistically significant differences in the early and late complication rates between ETGBD and EUS-GBD. The recurrence rate was $2.2 \%(23 / 1,030)$ vs. $3.0 \%$ (21/703), $p=0.352$. The bleeding complication rate was $0.65 \%(9 / 1,374)$ vs. $2.1 \%(16 / 749), p=0.005$. There were no statistically significant differences in safety and recurrence rate between ETGBD and EUS-GBD; however, the bleeding complication rate was significantly lower with ETGBD.

\section{DISCUSSION}

\section{Drainage in patients with acute cholecystitis}

In general, the first surgical treatment of choice for patients with acute cholecystitis is LC because of its radicality, safety, minimally invasive nature, and curability compared with OC. ${ }^{2,3,10}$ Ingraham et al. reported that the rates of complications, serious complications, and mortality at 30 days after the procedure were $3.1 \%, 1.4 \%$, and $0.27 \%$, respectively, in an analysis of 58,659 patients who underwent LC. ${ }^{69}$ Thus, some complications and a higher mortality rate may occur especially in elderly patients and/or in those with multiple severe comorbidities. In such patients with a high risk of emergency surgery, PTGBD has been widely performed as the first choice for internal gallbladder drainage. ${ }^{4}$ The technical success, clinical success, and complication rates of the PTGBD procedure were $98 \%, 90 \%$ and $3.7 \%$, respectively, indicating a relatively high technical success and reliable effectiveness. ${ }^{7}$ 


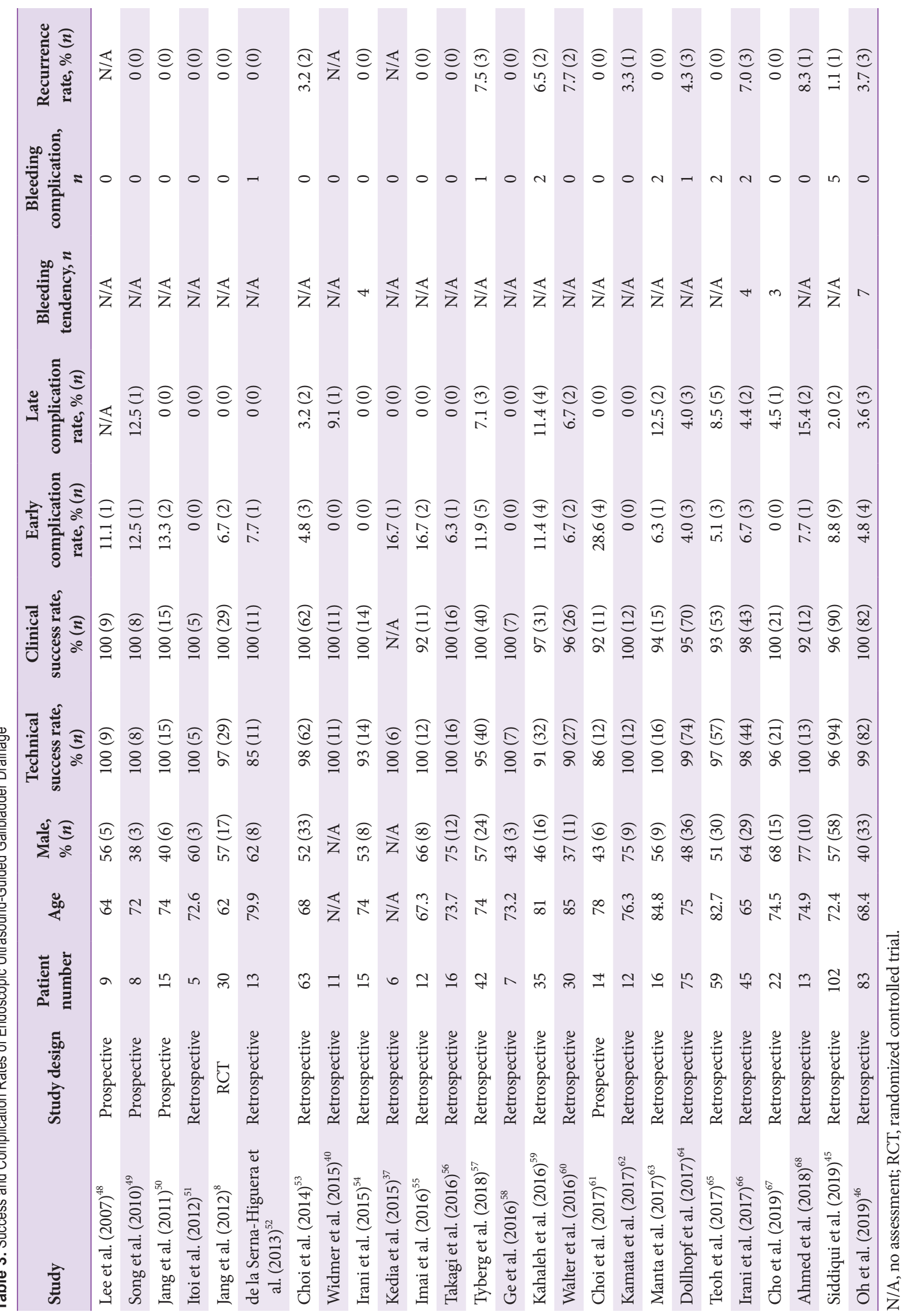


However, this method has complication and mortality rates as high as $14 \%$ and $17.5 \%$, respectively, in poor surgical candidates. ${ }^{6,70}$ In addition, PTGBD has several problematic features, such as a high recurrence rate (maximum 20\%), risk of self-stent removal because of postprocedural pain and discomfort, ${ }^{6,713,70}$ and fistula formation after the long-term placement and removal of a catheter. ${ }^{8}$ Moreover, PTGBD is contraindicated in patients with an anatomically inaccessible gallbladder, ascites, and thrombocytopenia. ${ }^{5}$ In such patients, EGBD is considered to be more useful as the second-line choice for internal drainage instead of surgery or PTGBD. ${ }^{5,10}$

Some studies reported that EGBD had a similar technical success rate to PTGBD but appeared to be safer and suitable for permanent placement because it has lower complication and recurrence rates than PTGBD. ${ }^{7,45,70}$ Accordingly, in our review, ETGBD and EUS-GBD showed relatively high technical and clinical success rates and low complication and recurrence rates. Considering the low recurrence rate of $\mathrm{EGBD},{ }^{33,45,61}$ long or permanent stent placement in EGBD could be possibly allowed. Therefore, EGBD may be considered as an alternative treatment to LC and PTGBD in various clinical situations.

\section{Current ATT management in the guidelines}

Antithrombotic agents have been increasingly used for preventing cardiovascular or cerebrovascular diseases in the aging population, and ATT involves the use of agents with 2 different mechanisms: antiplatelet therapy and anticoagulant therapy. Antiplatelet agents, such as aspirin and clopidogrel, are used for the prevention of cardiovascular and cerebrovascular diseases. Anticoagulants, such as warfarin and dabigatran, are used for preventing atrial fibrillation, deep vein thrombosis, and cardiac endoprostheses. ${ }^{71,72}$ Two types of anticoagulants are now increasingly used: direct-acting oral anticoagulants (DOACs) and non-vitamin K antagonist oral anticoagulants (NOACs). The risk of cardiovascular or cerebrovascular events associated with the discontinuation of antithrombotic agents should be fully considered when gallbladder drainage is performed. Recently, the prevention of thromboembolism has been considered to be more important than preventing bleeding complications owing to the severity and mortality of thromboembolism. Therefore, antiplatelet medications, at least aspirin monotherapy, should be continued in patients with a high risk of thromboembolism during therapeutic procedures. ${ }^{72}$

The management of antithrombotic agents for surgery, PTGBD, and EGBD is indicated in each guideline, considering the thrombosis risk of an individual patient and the procedural risk of bleeding. In patients undergoing elective LC, antiplatelet agents, at least aspirin monotherapy, should be continued if there is a high risk of thromboembolism. ${ }^{71,72} \mathrm{~Pa}$ tients receiving warfarin therapy should be switched to heparin bridging for 3-5 days, and those taking DOACs should stop the therapy for 1-2 days or receive heparin bridging therapy before surgery. ${ }^{72}$ The Society of Interventional Radiology ${ }^{73}$ recommends the discontinuation of clopidogrel 5 days before PTGBD; however, it is not essentially necessary to discontinue aspirin before the procedure in patients with a high risk of thromboembolism. In addition, it is suggested that the PTINR should be corrected to 1.5.

Guidelines from the Japan Gastroenterological Endoscopy Society $^{74}$ stipulate that low-bleeding-risk procedures can be performed without discontinuation of all types of antithrom-
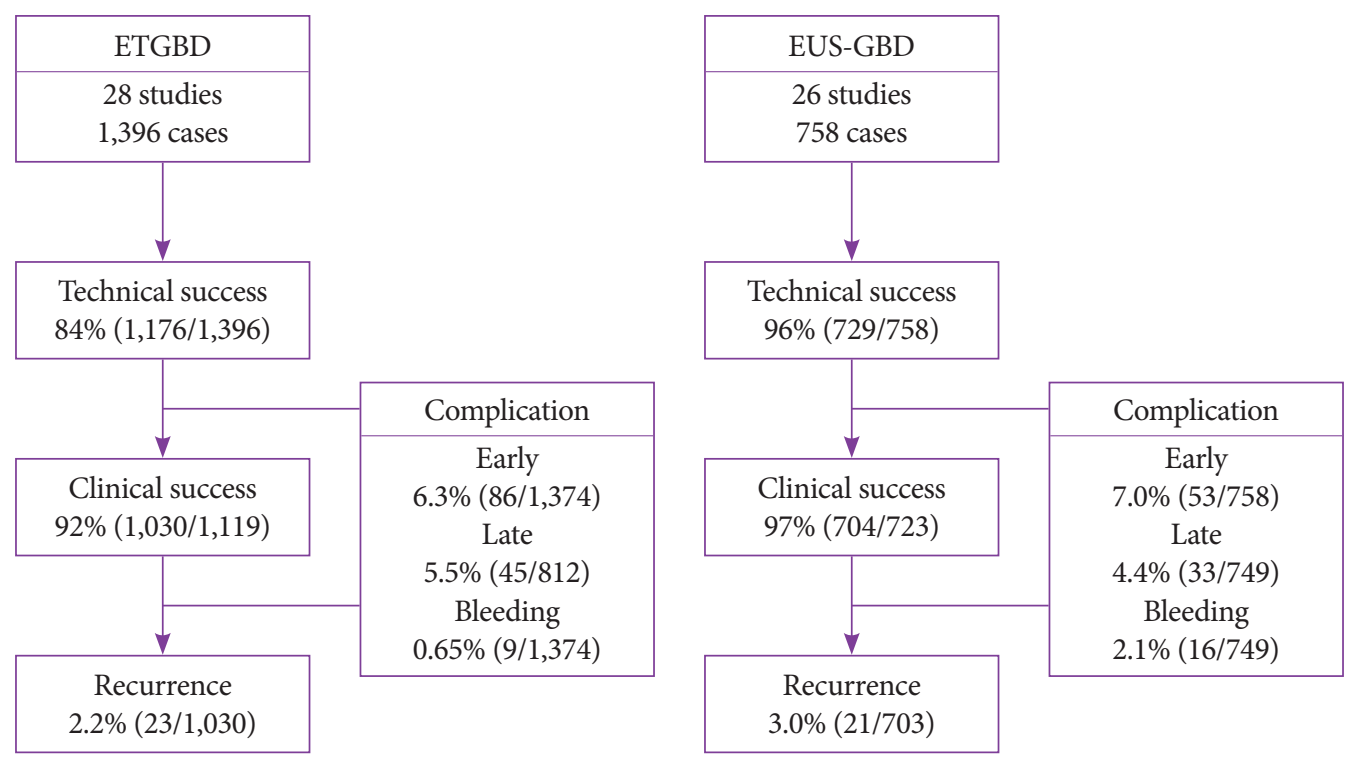

Fig. 1. Overall outcomes of endoscopic transpapillary gallbladder drainage (ETGBD) and endoscopic ultrasound-guided gallbladder drainage (EUS-GBD). 
botic agents, although procedures with a high bleeding risk should be carried out with discontinuation of antithrombotic agents. The guidelines also recommend substituting heparin bridging for warfarin or DOACs for these procedures, although the efficacy of bridging therapy is controversial, as Ono et al. reported that heparin bridging therapy increased the bleeding rates to approximately $20 \%{ }^{74}$ Further accumulation of evidence concerning the management of NOACs is required. ${ }^{74}$

EST and EUS-GBD are also classified as high-bleedingrisk procedures, ${ }^{74}$ and are allowed without discontinuation of aspirin monotherapy only in patients with a high risk of thromboembolism. However, ETGBD with EST in patients receiving aspirin monotherapy has the potential to increase the rate of bleeding complication. Therefore, ETGBD without EST may be more suitable for patients receiving ATT, as the discontinuation of ATT is not required.

\section{Current status of bleeding complication in ATT}

In general, the rate of bleeding complication after LC has been reported to be $0.1 \%-1.0 \%{ }^{2,71}$ On the other hand, the rate in patients receiving ATT was $0 \%-25.0 \% .^{11-18}$

Several studies reported that LC in patients receiving ATT might not increase bleeding-related events. ${ }^{12-17}$

On the contrary, other studies reported that emergency LC has a significant risk of bleeding complications in patients receiving mono- or multiple $\mathrm{ATT}^{17,18}$ and in those receiving heparin bridging therapy. ${ }^{11}$ In our review, $6.5 \%$ of the patients receiving ATT who underwent LC (OC in part) experienced bleeding complications, and the rate was significantly higher than that in patients without ATT. Thus, the use of LC in patients receiving ATT remains controversial.

With respect to PTGBD, 2 studies concluded that there was no significant increase in bleeding complications in patients receiving ATT and/or with coagulopathy. ${ }^{19,20}$ On the other hand, another study concluded that continuation of antiplatelet agents could increase the risk of severe bleeding after $\mathrm{PTBD}^{21}{ }^{21}$ The use of PTGBD in patients receiving ATT also remains controversial, as this technique may pose a bleeding risk in patients with acute cholecystitis receiving ATT.

In our review, the bleeding complication rate of ETGBD was low $(0.65 \%)$ in 1,374 patients, and only 1 patient $(0.5 \%)$ experienced bleeding after the procedure among 191 patients receiving ATT or with some type of coagulopathy. EST was considered a main cause of bleeding complications after $\mathrm{ERCP}^{74}$ as 151 patients who underwent ETGBD without EST did not show any bleeding complications. In EUS-GBD, the rate of bleeding complication was $2.1 \%$. Among patients receiving ATT or with coagulopathy, bleeding complication was confirmed in $2.4 \%$ (18 of 758 patients); in 2 patients, bleeding might have been caused by ATT. Some studies reported that EUS-GBD could be safer to perform than PTGBD in patients with anticoagulant therapy because the gastrointestinal tract is less vascular than the liver. ${ }^{13,58}$ However, on the basis of our review, bleeding complications might occur with a certain probability, although they are rarely severe and fatal..$^{63}$

\section{Future perspectives in EGBD for ATT}

ETGBD may be safer than EUS-GBD or PTGBD in patients receiving ATT or with coagulopathy, because this procedure

Table 4. Assessment of the Review Results Regarding a Comparison with the Recommendation of the Guideline in Patients with Antithrombotic Therapy including Bleeding Tendency

\begin{tabular}{|c|c|c|c|}
\hline & Guidelines & Our review & Assessment \\
\hline $\begin{array}{l}\text { Surgery } \\
\text { (mainly LC) }\end{array}$ & $\begin{array}{l}\text { 1) Discontinuation of ATT is recommended. } \\
\text { 2) Continuation of aspirin monotherapy is } \\
\text { acceptable. }\end{array}$ & $\begin{array}{l}\text { 1) Surgery under continuation of ATT is } \\
\text { controversial. } \\
\text { 2) Bleeding complication rate is } 6.5 \% \text { in patients } \\
\text { receiving ATT. }\end{array}$ & $\begin{array}{l}\text { Need further } \\
\text { discussion }\end{array}$ \\
\hline PTGBD & $\begin{array}{l}\text { 1) Discontinuation of ATT is recommended. } \\
\text { 2) Continuation of aspirin monotherapy is } \\
\text { acceptable. }\end{array}$ & $\begin{array}{l}\text { 1) PTGBD under continuation of ATT is } \\
\text { controversial. }\end{array}$ & $\begin{array}{l}\text { Need further } \\
\text { discussion }\end{array}$ \\
\hline ETGBD & $\begin{array}{l}\text { 1) With EST: discontinuation of ATT is } \\
\text { recommended. } \\
\text { 2) Without EST: continuation of all type ATT } \\
\text { is recommended. }\end{array}$ & $\begin{array}{l}\text { 1) Few evidence } \\
\text { 2) Bleeding complication rate is } 0.65 \% \text { in all } \\
\text { patients, } 0 \% \text { in all patients without EST, } 0.5 \% \\
\text { in patients with bleeding tendency. }\end{array}$ & Recommendation \\
\hline EUS-GBD & 1) Discontinuation of ATT is recommended. & $\begin{array}{l}\text { 1) Few evidence } \\
\text { 2) Bleeding complication rate } 2.1 \% \text { in all patients, } \\
11.1 \% \text { in patients with bleeding tendency. }\end{array}$ & $\begin{array}{l}\text { Need further } \\
\text { discussion }\end{array}$ \\
\hline
\end{tabular}

ATT, antithrombotic therapy; EST, endoscopic sphincterotomy; ETGBD, endoscopic transpapillary gallbladder drainage; EUS-GBD, endoscopic ultrasound-guided gallbladder drainage; LC, laparoscopic cholecystectomy; PTGBD, percutaneous transhepatic gallbladder drainage. 
does not require needle puncture or fistula dilation. ${ }^{51}$ However, the technical success rate of ETGBD is lower than that of EUS-GBD. ETGBD is technically challenging, especially the cannulation procedure, ${ }^{40,70}$ and requires an expert endoscopist. More experience with wire manipulation might improve the technical success rates. ${ }^{29}$ In addition, special methods combined with cholangioscopy have been proposed, and their superiority in detecting the orifice of the cystic duct in the common bile duct has been proven. ${ }^{38}$ Improvements in the technique and modality could further increase the success rate.

From the result of the present review, we conclude that ETGBD without EST may be an ideal treatment for patients receiving ATT from the viewpoint of preventing bleeding complications. However, patients undergoing ETGBD without EST may have a higher risk of other common complications, such as pancreatitis and cholangitis, different from EUS-GBD. Lee et al. ${ }^{33}$ emphasized the risk of post-EST bleeding and that EST should not be necessarily required in all cases. Thus, EST should be avoided whenever possible to reduce the risk of severe complications. Endoscopic papillary balloon dilation (EPBD) may solve this dilemma, because this technique is classified as a low-bleeding-risk procedure based on its lower risk of bleeding complications than $\mathrm{EST} .^{74}$ In addition, EPBD prevents complications such as pancreatitis or cholangitis resulting from the obstruction of the papillary orifice..$^{33}$ Therefore, ETGBD with on-demand EPBD should be alternatively performed in patients with acute cholecystitis with coagulopathy, cirrhotic liver, and ATT. EUS-GBD may be the second ideal option for drainage treatment when ETGBD has failed or is not adopted. The results of the present review are summarized and compared with the guideline recommendations in Table 4.

This review has some limitations. First, the sample size is relatively small for evaluating the incidence and risk of relatively rare bleeding complications. In future studies, a much higher number of samples may be possible. Second, as the number of patients receiving antithrombotic agents may also be small, the comparisons of the different drainage methods are partly indirect. Third, the baseline characteristics of patients with ATT and coagulopathy that possibly influenced the review results were not fully known. However, we believe that the scale of their influence did not cause a serious misunderstanding in the review process.

\section{CONCLUSIONS}

The use of LC and PTGBD in patients with acute cholecystitis receiving ATT or with coagulopathy remains controver- sial. Therefore, ETGBD without EST should be considered as the first-choice treatment in patients with acute cholecystitis who cannot undergo cholecystectomy because of the use of ATT.

In addition, EUS-GBD may also be a potential alternative treatment in patients who are not suitable for PTGBD and ETGBD. To clarify the efficacy and feasibility of EGBD in patients receiving ATT, further studies, especially randomized controlled prospective studies, are needed.

\section{Conflicts of Interest}

The authors have no financial conflict of interest.

\section{Author Contributions}

Conceptualization: Ryota Sagami, Kenji Hayasaka, Hideaki Harada Data curation: RS, Hidefumi Nishikiori

Formal analysis: RS, Yuji Amano

Investigation: RS

Methodology: RS

Project administration: RS

Resources: RS

Software: $\mathrm{HH}$

Supervision: YA

Validation: YA

Visualization: YA

Writing-original draft: RS

Writing-review\&editing: YA

ORCID

Kenji Hayasaka: https://orcid.org/0000-0002-1372-9701

Hidefumi Nishikiori: https://orcid.org/0000-0003-1767-8996

Hideaki Harada: https://orcid.org/0000-0002-9951-7100

Yuji Amano: https://orcid.org/0000-0002-4480-3222

\section{REFERENCES}

1. Yokoe M, Hata J, Takada T, et al. Tokyo guidelines 2018: diagnostic criteria and severity grading of acute cholecystitis (with videos). J Hepatobiliary Pancreat Sci 2018;25:41-54.

2. McMahon AJ, Fischbacher CM, Frame SH, MacLeod MC. Impact of laparoscopic cholecystectomy: a population-based study. Lancet 2000;356:1632-1637.

3. Hannan EL, Imperato PJ, Nenner RP, Starr H. Laparoscopic and open cholecystectomy in New York State: mortality, complications, and choice of procedure. Surgery 1999;125:223-231.

4. Patel M, Miedema BW, James MA, Marshall JB. Percutaneous cholecystostomy is an effective treatment for high-risk patients with acute cholecystitis. Am Surg 2000;66:33-37.

5. Itoi T, Sofuni A, Itokawa F, et al. Endoscopic transpapillary gallbladder drainage in patients with acute cholecystitis in whom percutaneous transhepatic approach is contraindicated or anatomically impossible (with video). Gastrointest Endosc 2008;68:455-460.

6. Tamada K, Seki H, Sato K, et al. Efficacy of endoscopic retrograde cholecystoendoprosthesis (ERCCE) for cholecystitis. Endoscopy 1991;23:2-3.

7. Itoi T, Coelho-Prabhu N, Baron TH. Endoscopic gallbladder drainage for management of acute cholecystitis. Gastrointest Endosc 2010;71:1038-1045

8. Jang JW, Lee SS, Song TJ, et al. Endoscopic ultrasound-guided transmu- 
ral and percutaneous transhepatic gallbladder drainage are comparable for acute cholecystitis. Gastroenterology 2012;142:805-811.

9. Baron TH, Topazian MD. Endoscopic transduodenal drainage of the gallbladder: implications for endoluminal treatment of gallbladder disease. Gastrointest Endosc 2007;65:735-737.

10. Mori Y, Itoi T, Baron TH, et al. Tokyo guidelines 2018: management strategies for gallbladder drainage in patients with acute cholecystitis (with videos). J Hepatobiliary Pancreat Sci 2018;25:87-95.

11. Ercan M, Bostanci EB, Ozer I, et al. Postoperative hemorrhagic complications after elective laparoscopic cholecystectomy in patients receiving long-term anticoagulant therapy. Langenbecks Arch Surg 2010;395:247253.

12. Ono K, Idani H, Hidaka H, Kusudo K, Koyama Y, Taguchi S. Effect of aspirin continuation on blood loss and postoperative morbidity in patients undergoing laparoscopic cholecystectomy or colorectal cancer resection. Surg Laparosc Endosc Percutan Tech 2013;23:97-100.

13. Noda T, Hatano H, Dono K, et al. Safety of early laparoscopic cholecystectomy for patients with acute cholecystitis undergoing antiplatelet or anticoagulation therapy: a single-institution experience. Hepatogastroenterology 2014;61:1501-1506.

14. Anderson K, Jupiter DC, Abernathy SW, Frazee RC. Should clopidogrel be discontinued before laparoscopic cholecystectomy? Am J Surg 2014;208:926-931; discussion 930-931.

15. Joseph B, Rawashdeh B, Aziz H, et al. An acute care surgery dilemma: emergent laparoscopic cholecystectomy in patients on aspirin therapy. Am J Surg 2015;209:689-694.

16. Akahoshi K, Ochiai T, Takaoka A, et al. Emergency cholecystectomy for patients on antiplatelet therapy. Am Surg 2017;83:486-490.

17. Yun JH, Jung HI, Lee HU, Baek MJ, Bae SH. The efficacy of laparoscopic cholecystectomy without discontinuation in patients on antithrombotic therapy. Ann Surg Treat Res 2017;92:143-148.

18. Kawamoto Y, Fujikawa T, Sakamoto Y, et al. Effect of antithrombic therapy on bleeding complications in patients receiving emergency cholecystectomy for acute cholecystitis. J Hepatobiliary Pancreat Sci 2018;25:518-526.

19. Dewhurst C, Kane RA, Mhuircheartaigh JN, Brook O, Sun M, Siewert B. Complication rate of ultrasound-guided percutaneous cholecystostomy in patients with coagulopathy. AJR Am J Roentgenol 2012;199:W753-W760.

20. Shibasaki S, Takahashi N, Toi H, et al. Percutaneous transhepatic gallbladder drainage followed by elective laparoscopic cholecystectomy in patients with moderate acute cholecystitis under antithrombotic therapy. J Hepatobiliary Pancreat Sci 2014;21:335-342.

21. Hamada T, Yasunaga H, Nakai Y, et al. Severe bleeding after percutaneous transhepatic drainage of the biliary system: effect of antithrombotic agents--analysis of 34606 cases from a Japanese nationwide administrative database. Radiology 2015;274:605-613.

22. Feretis C, Apostolidis N, Mallas E, Manouras A, Papadimitriou J. Endoscopic drainage of acute obstructive cholecystitis in patients with increased operative risk. Endoscopy 1993;25:392-395.

23. Johlin FC Jr, Neil GA. Drainage of the gallbladder in patients with acute acalculous cholecystitis by transpapillary endoscopic cholecystotomy. Gastrointest Endosc 1993;39:645-651.

24. Nakatsu T, Okada H, Saito K, et al. Endoscopic transpapillary gallbladder drainage (ETGBD) for the treatment of acute cholecystitis. J Hepatobiliary Pancreat Sci 1997;4:31-35.

25. Shrestha R, Trouillot TE, Everson GT. Endoscopic stenting of the gallbladder for symptomatic gallbladder disease in patients with end-stage liver disease awaiting orthotopic liver transplantation. Liver Transpl Surg 1999;5:275-281.

26. Conway JD, Russo MW, Shrestha R. Endoscopic stent insertion into the gallbladder for symptomatic gallbladder disease in patients with endstage liver disease. Gastrointest Endosc 2005;61:32-36.

27. Toyota N, Takada T, Amano H, Yoshida M, Miura F, Wada K. Endoscopic naso-gallbladder drainage in the treatment of acute cholecystitis: alleviates inflammation and fixes operator's aim during early laparoscopic cholecystectomy. J Hepatobiliary Pancreat Surg 2006;13:80-85.

28. Schlenker C, Trotter JF, Shah RJ, et al. Endoscopic gallbladder stent placement for treatment of symptomatic cholelithiasis in patients with end-stage liver disease. Am J Gastroenterol 2006;101:278-283.

29. Kjaer DW, Kruse A, Funch-Jensen P. Endoscopic gallbladder drainage of patients with acute cholecystitis. Endoscopy 2007;39:304-308.

30. Ogawa O, Yoshikumi H, Maruoka N, et al. Predicting the success of endoscopic transpapillary gallbladder drainage for patients with acute cholecystitis during pretreatment evaluation. Can J Gastroenterol 2008;22:681-685.

31. Pannala R, Petersen BT, Gostout CJ, Topazian MD, Levy MJ, Baron TH. Endoscopic transpapillary gallbladder drainage: 10-year single center experience. Minerva Gastroenterol Dietol 2008;54:107-113.

32. Mutignani M, Iacopini F, Perri V, et al. Endoscopic gallbladder drainage for acute cholecystitis: technical and clinical results. Endoscopy 2009;41:539-546.

33. Lee TH, Park DH, Lee SS, et al. Outcomes of endoscopic transpapillary gallbladder stenting for symptomatic gallbladder diseases: a multicenter prospective follow-up study. Endoscopy 2011;43:702-708.

34. Maekawa S, Nomura R, Murase T, Ann Y, Oeholm M, Harada M. Endoscopic gallbladder stenting for acute cholecystitis: a retrospective study of 46 elderly patients aged 65 years or older. BMC Gastroenterol 2013;13:65.

35. Yane K, Maguchi H, Katanuma A, et al. Feasibility, efficacy, and predictive factors for the technical success of endoscopic nasogallbladder drainage: a prospective study. Gut Liver 2015;9:239-246.

36. Itoi $\mathrm{T}$, Kawakami H, Katanuma A, et al. Endoscopic nasogallbladder tube or stent placement in acute cholecystitis: a preliminary prospective randomized trial in Japan (with videos). Gastrointest Endosc 2015;81:111-118.

37. Kedia P, Sharaiha RZ, Kumta NA, et al. Endoscopic gallbladder drainage compared with percutaneous drainage. Gastrointest Endosc 2015;82:1031-1036.

38. McCarthy ST, Tujios S, Fontana RJ, et al. Endoscopic transpapillary gallbladder stent placement is safe and effective in high-risk patients without cirrhosis. Dig Dis Sci 2015;60:2516-2522.

39. Tujios SR, Rahnama-Moghadam S, Elmunzer JB, et al. Transpapillary gallbladder stents can stabilize or improve decompensated cirrhosis in patients awaiting liver transplantation. J Clin Gastroenterol 2015;49:771777.

40. Widmer J, Alvarez P, Sharaiha RZ, et al. Endoscopic gallbladder drainage for acute cholecystitis. Clin Endosc 2015;48:411-420.

41. Hatanaka T, Itoi T, Ijima M, et al. Efficacy and safety of endoscopic gallbladder stenting for acute cholecystitis in patients with concomitant unresectable cancer. Intern Med 2016;55:1411-1417.

42. Inoue T, Okumura F, Kachi K, et al. Long-term outcomes of endoscopic gallbladder stenting in high-risk surgical patients with calculous cholecystitis (with videos). Gastrointest Endosc 2016;83:905-913.

43. Yang MJ, Yoo BM, Kim JH, et al. Endoscopic naso-gallbladder drainage versus gallbladder stenting before cholecystectomy in patients with acute cholecystitis and a high suspicion of choledocholithiasis: a prospective randomised preliminary study. Scand J Gastroenterol 2016;51:472-478.

44. Itoi T, Takada T, Hwang TL, et al. Percutaneous and endoscopic gallbladder drainage for acute cholecystitis: international multicenter comparative study using propensity score-matched analysis. J Hepatobiliary Pancreat Sci 2017;24:362-368.

45. Siddiqui A, Kunda R, Tyberg A, et al. Three-way comparative study of endoscopic ultrasound-guided transmural gallbladder drainage using lumen-apposing metal stents versus endoscopic transpapillary drainage versus percutaneous cholecystostomy for gallbladder drainage in highrisk surgical patients with acute cholecystitis: clinical outcomes and success in an international, multicenter study. Surg Endosc 2019;33:12601270. 
46. Oh D, Song TJ, Cho DH, et al. EUS-guided cholecystostomy versus endoscopic transpapillary cholecystostomy for acute cholecystitis in highrisk surgical patients. Gastrointest Endosc 2019;89:289-298.

47. Iino C, Shimoyama T, Igarashi T, et al. Comparable efficacy of endoscopic transpapillary gallbladder drainage and percutaneous transhepatic gallbladder drainage in acute cholecystitis. Endosc Int Open 2018;6:E594-E601.

48. Lee SS, Park DH, Hwang CY, et al. EUS-guided transmural cholecystostomy as rescue management for acute cholecystitis in elderly or high-risk patients: a prospective feasibility study. Gastrointest Endosc 2007;66:1008-1012.

49. Song TJ, Park DH, Eum JB, et al. EUS-guided cholecystoenterostomy with single-step placement of a 7F double-pigtail plastic stent in patients who are unsuitable for cholecystectomy: a pilot study (with video). Gastrointest Endosc 2010;71:634-640.

50. Jang JW, Lee SS, Park DH, Seo DW, Lee SK, Kim MH. Feasibility and safety of EUS-guided transgastric/transduodenal gallbladder drainage with single-step placement of a modified covered self-expandable metal stent in patients unsuitable for cholecystectomy. Gastrointest Endosc 2011;74:176-181.

51. Itoi T, Binmoeller KF, Shah J, et al. Clinical evaluation of a novel lumen-apposing metal stent for endosonography-guided pancreatic pseudocyst and gallbladder drainage (with videos). Gastrointest Endosc 2012;75:870-876.

52. de la Serna-Higuera C, Pérez-Miranda M, Gil-Simón P, et al. EUS-guided transenteric gallbladder drainage with a new fistula-forming, lumen-apposing metal stent. Gastrointest Endosc 2013;77:303-308.

53. Choi JH, Lee SS, Choi JH, et al. Long-term outcomes after endoscopic ultrasonography-guided gallbladder drainage for acute cholecystitis. Endoscopy 2014;46:656-661.

54. Irani S, Baron TH, Grimm IS, Khashab MA. EUS-guided gallbladder drainage with a lumen-apposing metal stent (with video). Gastrointest Endosc 2015;82:1110-1115.

55. Imai $\mathrm{H}$, Kitano $\mathrm{M}$, Omoto $\mathrm{S}$, et al. EUS-guided gallbladder drainage for rescue treatment of malignant distal biliary obstruction after unsuccessful ERCP. Gastrointest Endosc 2016;84:147-151.

56. Takagi W, Ogura T, Sano T, et al. EUS-guided cholecystoduodenostomy for acute cholecystitis with an anti-stent migration and anti-food impaction system; a pilot study. Therap Adv Gastroenterol 2016;9:19-25.

57. Tyberg A, Saumoy M, Sequeiros EV, et al. EUS-guided versus percutaneous gallbladder drainage: isn't it time to convert? J Clin Gastroenterol 2018;52:79-84.

58. Ge N, Sun S, Sun S, Wang S, Liu X, Wang G. Endoscopic ultrasound-assisted transmural cholecystoduodenostomy or cholecystogastrostomy as a bridge for per-oral cholecystoscopy therapy using double-flanged fully covered metal stent. BMC Gastroenterol 2016;16:9.

59. Kahaleh M, Perez-Miranda M, Artifon EL, et al. International collaborative study on EUS-guided gallbladder drainage: are we ready for prime time? Dig Liver Dis 2016;48:1054-1057.

60. Walter D, Teoh AY, Itoi T, et al. EUS-guided gall bladder drainage with a lumen-apposing metal stent: a prospective long-term evaluation. Gut 2016;65:6-8.

61. Choi JH, Kim HW, Lee JC, et al. Percutaneous transhepatic versus
EUS-guided gallbladder drainage for malignant cystic duct obstruction. Gastrointest Endosc 2017;85:357-364.

62. Kamata K, Takenaka M, Kitano M, et al. Endoscopic ultrasound-guided gallbladder drainage for acute cholecystitis: long-term outcomes after removal of a self-expandable metal stent. World J Gastroenterol 2017;23:661-667.

63. Manta R, Zulli C, Zullo A, et al. Endoscopic ultrasound-guided gallbladder drainage for acute cholecystitis with a silicone-covered nitinol short bilaterally flared stent: a case series. Endosc Int Open 2017;5:E1111-E1115.

64. Dollhopf M, Larghi A, Will U, et al. EUS-guided gallbladder drainage in patients with acute cholecystitis and high surgical risk using an electrocautery-enhanced lumen-apposing metal stent device. Gastrointest Endosc 2017;86:636-643.

65. Teoh AYB, Serna C, Penas I, et al. Endoscopic ultrasound-guided gallbladder drainage reduces adverse events compared with percutaneous cholecystostomy in patients who are unfit for cholecystectomy. Endoscopy 2017;49:130-138.

66. Irani S, Ngamruengphong S, Teoh A, et al. Similar efficacies of endoscopic ultrasound gallbladder drainage with a lumen-apposing metal stent versus percutaneous transhepatic gallbladder drainage for acute cholecystitis. Clin Gastroenterol Hepatol 2017;15:738-745.

67. Cho DH, Jo SJ, Lee JH, et al. Feasibility and safety of endoscopic ultrasound-guided gallbladder drainage using a newly designed lumen-apposing metal stent. Surg Endosc 2019;33:2135-2141.

68. Ahmed O, Ogura T, Eldahrouty A, et al. Endoscopic ultrasound-guided gallbladder drainage: results of long-term follow-up. Saudi J Gastroenterol 2018;24:183-188.

69. Ingraham AM, Cohen ME, Ko CY, Hall BL. A current profile and assessment of North American cholecystectomy: results from the American college of surgeons national surgical quality improvement program. J Am Coll Surg 2010;211:176-186.

70. Khan MA, Atiq O, Kubiliun N, et al. Efficacy and safety of endoscopic gallbladder drainage in acute cholecystitis: is it better than percutaneous gallbladder drainage? Gastrointest Endosc 2017;85:76-87.e3.

71. Fujikawa T, Ando K. Safety of laparoscopic surgery in digestive diseases with special reference to antithrombotic therapy: a systematic review of the literature. World J Clin Cases 2018;6:767-775.

72. Korte W, Cattaneo M, Chassot PG, et al. Peri-operative management of antiplatelet therapy in patients with coronary artery disease: joint position paper by members of the working group on Perioperative Haemostasis of the Society on Thrombosis and Haemostasis Research (GTH), the working group on Perioperative Coagulation of the Austrian Society for Anesthesiology, Resuscitation and Intensive Care (OGARI) and the Working Group Thrombosis of the European Society for Cardiology (ESC). Thromb Haemost 2011;105:743-749.

73. Patel IJ, Davidson JC, Nikolic B, et al. Consensus guidelines for periprocedural management of coagulation status and hemostasis risk in percutaneous image-guided interventions. J Vasc Interv Radiol 2012;23:727736.

74. Ono S, Fujishiro M, Ikeda Y, Komuro I, Koike K. Recent clinical management of antithrombotic agents for gastrointestinal endoscopy after revision of guidelines in Japan. Dig Endosc 2015;27:649-656. 